\title{
Relationship between surface dipole, work function and charge transfer: Some exceptions to an established rule
}

\author{
T. C. Leung, C. L. Kao, and W. S. Su \\ Department of Physics, National Chung Cheng University, Taiwan, Republic of China \\ Y. J. Feng and C. T. Chan \\ Department of Physics, Hong Kong University of Science and Technology, Clear Water Bay, Hong Kong, China
}

(Received 26 May 2003; revised manuscript received 18 August 2003; published 17 November 2003)

Local-density-functional calculations are used to study the change of work functions induced by a layer of adsorbates. We investigated and compared the work function of a monolayer of $\mathrm{Mo}, \mathrm{Ag}, \mathrm{Au}, \mathrm{Fe}, \mathrm{Co}, \mathrm{Ni}, \mathrm{Nb}$, $\mathrm{Li}, \mathrm{N}$, and $\mathrm{O}$ on $\mathrm{W}(100), \mathrm{W}(110), \mathrm{W}(211)$, and $\mathrm{W}(111)$ surfaces. While many systems obey the commonly accepted rule that electronegative adsorbates increase the work function of the surface, we find some exceptions. For example, overlayers of $\mathrm{Fe}, \mathrm{Co}$, and $\mathrm{Ni}$ increase the work function of W(100), W(211), and W(111), but decrease the work function of the $\mathrm{W}(110)$ surface, although the charge transfer is the same in all orientations. We found that even a layer of oxygen can decrease the work function of W(100), although there are always electrons transferred from the $\mathrm{W}$ substrate to the oxygen adsorbates. In order to understand these results, we established the relationship between surface dipole density and work function within the framework of local-density formalism. It turns out that subtle details of the charge transfer can determine the sign and magnitude of surface dipole change, leading to a strong dependence on the orientation of the substrate, with the consequence that the work-function changes are not always governed by the sign and quantity of adsorbate induced charge transfer.

DOI: 10.1103/PhysRevB.68.195408

PACS number(s): 73.30. $+\mathrm{y}, 73.20 .-\mathrm{r}$

\section{INTRODUCTION}

Work function is one of the most fundamental electronic properties of a metallic surface. It measures the minimum energy required to extract an electron from the inside of a bulk solid to the outside. It is defined more precisely as the energy difference between the state in which an electron has been removed to a point sufficiently far outside the surface so that the image force is negligible and the state in which the electron is in the bulk solid. In general, the work function for each face of a monocrystalline sample differs by amounts ranging from one-tenth to half an $\mathrm{eV}$. Wigner and Bardeen ${ }^{1}$ proposed that the work function is given by

$$
\phi=-\mu+D=-\mu-\frac{e p}{\varepsilon_{0}} .
$$

The first term is the bulk chemical potential $\mu$ of the electrons relative to the mean electrostatic potential on the metal interior, and the second term corresponds to the energy necessary to penetrate the dipole barrier $D$ at the surface. The surface dipole barrier, which is responsible for the anisotropy of the work function of metals, is formed by the redistribution of the electron density on the surface. For a jellium surface, electrons spilling out into the vacuum forms a negative dipole layer. ${ }^{2}$ For a crystal with atomic structure, consider a surface that is exposed by splitting the crystal into two. If there is no change in the charge distribution of the surface atoms, then there would be no dipole barrier. Smoluchowski ${ }^{3}$ suggested there are two effects on the dipole layer: the first is a partial spread of the charge out of the polyhedra of the surface atoms and the second is a tendency to smooth out the surface of the polyhedra. The first effect forms a negative dipole layer which increases the work function, and the second effect forms a positive dipole layer which reduces the work function. Since the two effects are comparable in magnitude, it is difficult to predict the magnitude of the surface dipole without numerical computations. However, it is generally believed that the change of the work function of a surface as affected by a layer of adsorbates can be predicted by knowing the electronegativity of the adsorbates. If the adsorbate is more electronegative than the substrate, electrons will be transferred to the adsorbate layer, causing an excess of negative charges on the outside and an excess of positive charges on the inside of the surface. This leads to a negative dipole (pointing inward) that reinforces the original surface dipole due to electron "spill out," causing the work function to increase. On the contrary, a decrease in work function is expected for electropositive adsorbates. Well-known examples are $\mathrm{Cl}$ on $\mathrm{Cu}(111),{ }^{4}$ in which an electronegative adsorbate increases the substrate work function, and $\mathrm{Li}$ on $\mathrm{W}(111),{ }^{5}$ where the work function of the substrate is reduced by an electropositive adsorbate.

We will show in the present paper that this picture is only approximately correct; exceptions can be found. The original intention of this study is to explore the effect of ultrathin layers of certain adsorbate layers on refractory metal tips as field emitters, and the work function is one of the key factors that affect field emission. We found that some of the results for specific systems do not follow the notion that "electronegative adsorbates increase work function," and this prompted us to study the relationship between charge transfer and work-function change more systematically, using a fairly large number of adsorbates of different electronegativities and we considered different substrate orientations. Specifically, we investigated and compared the work function of a 
monolayer of $\mathrm{Mo}, \mathrm{Ag}, \mathrm{Au}, \mathrm{Fe}, \mathrm{Co}, \mathrm{Ni}, \mathrm{Nb}, \mathrm{Li}, \mathrm{N}$, and $\mathrm{O}$ adsorbed on W(100), W(110), W(211), and W(111) surfaces. The unit cells are all $p(1 \times 1)$. All the systems are force relaxed so that they are in a configuration corresponding to local minima of energy surfaces, but some of these systems are not necessarily in the global minima in energy as far as coverage, adsorption site, or magnetic state is concerned. This should not affect us here since our purpose is to use a variety of systems to relate the adsorbate induced charge transfer to the change of surface dipole and work function, and not to study chemisorption thermodynamics of a specific system. Our conclusions are drawn on local-density functional calculations. Quantities such as the charge density and the Coulomb potential are directly accessible from the localdensity-functional calculations, and these information allow us to calculate the value of the dipole moment of the dipole layer and the work function.

Local-density formalism is not an exact theory, but can be viewed as a very useful parameter-free formalism to investigate the relationship we are looking for. It is well documented that local-density formalism gives good ground-state properties of a wide variety of systems, ${ }^{6}$ and often gives work function to the order of $0.1 \mathrm{eV}$ when compared with experiments. ${ }^{7}$ The present calculation is perhaps the most systematic study on the effect of adsorbate on work function of the metal surfaces using first-principles calculations.

\section{METHOD OF CALCULATION}

The calculations are done within the framework of localdensity approximation (LDA) (Ref. 8) using the Vienna $a b$ initio simulation package ${ }^{9}$ and ultrasoft pseudopotentials. ${ }^{10}$ We used the standard slab geometry to model the surface systems. The $\mathrm{W}$ substrates are modeled by 11 layer slabs for the $\mathrm{W}(100), \mathrm{W}(110)$, and $\mathrm{W}(211)$ orientations, and 21 layer slabs are used for $\mathrm{W}(111)$. The surface unit cells are $p(1$ $\times 1$ ). The overlayers are pseudomorphic and are put on both sides of the substrate $\mathrm{W}$ slab. All calculations assume no spin polarization. The vacuum thickness is $10 \AA$. The adsorption sites are taken to be that of the continuation of the substrate, and all atomic coordinates are fully relaxed. We note again that the sites may not be thermodynamically most stable for a particular adsorbate, but they do serve as prototypical systems to study the relationship between charge transfer and work-function changes. For the case of the (111) surface, the overlayer is a "geometric monolayer," which is $1 / 3$ of a "physical monolayer," 11 a term used in the literature to describe the number of atoms needed to shadow all the substrate atoms in a ball-and-stick model projected onto the surface.

We will first find the relationship between work function and surface dipole density with the LDA formulation. Since the dipole barrier can only depend on $z$ by symmetry, we can use $\rho(z)$, which is the charge density at $z$ averaged over the xy plane, instead of the full position dependent $\rho(\mathbf{r})$, without loss of generality. This plane averaged $\rho(z)$ is given by

$$
\rho(z)=\frac{1}{A} \int_{0}^{a_{1}} d x \int_{0}^{a_{2}} d y \rho(x, y, z),
$$

where $A$ is the area of the surface unit cell. Using Poisson's equation, the dipole moment density of the dipole layer is given by

$$
\begin{aligned}
p= & \int_{z_{0}}^{c / 2} z \rho_{t}(z) d z=\frac{-\varepsilon_{0}}{A} \int_{0}^{a_{1}} d x \int_{0}^{a_{2}} d y \\
& \times \int_{z_{0}}^{c / 2} d z\left[\frac{\partial^{2} V}{\partial x^{2}}+\frac{\partial^{2} V}{\partial y^{2}}+\frac{\partial^{2} V}{\partial z^{2}}\right] z,
\end{aligned}
$$

where $\rho_{t}=\rho_{\text {electron }}+\rho_{\text {ion }}$ is the total charge density averaged over the $x y$ plane, which includes the contribution from the electrons $\left(\rho_{\text {electron }}\right)$ and ions $\left(\rho_{\text {ion }}\right)$, at some position $z$. In our calculations, we will use the convention that the center of the slab is chosen to be at $z=0$, then $c / 2$ corresponds to the center of the vacuum of the supercell. The starting point $z_{0}$ can be chosen to be the mid point between any two layers that are deep in the bulk. The first two terms in right-hand side of Eq. (3) vanish inside the bracket due to periodicity. By the symmetry of the system, the value of $\partial V / \partial z$ is equal to zero at $c / 2$ and $z_{0}$. If we define the $x y$ plane averaged Coulomb potential $V(z)$ as

$$
V(z)=\frac{1}{A} \int_{0}^{a_{1}} d x \int_{0}^{a_{2}} d y V(x, y, z),
$$

we get a simple relation between the dipole density and the change of the $x y$ plane averaged Coulomb potential as follows:

$$
\frac{p}{\varepsilon_{0}}=\int_{z_{0}}^{c / 2} d V(z)=V(c / 2)-V\left(z_{0}\right) .
$$

If the value of the Fermi level, $E_{f}$, is set equal to zero, the value of $-e V(c / 2)$ is just equal to the value of the work function. Therefore, Eq. (5) can be rewritten as

$$
\phi=-e V\left(z_{0}\right)-\frac{e p}{\varepsilon_{0}} .
$$

If we choose the unit such that the unit of length is in terms of angstrom, the unit of charge is in terms of the number of electrons, and the unit of energy is in terms of eV, Eq. (6) can be written as

$$
\phi=-e V\left(z_{0}\right)-180.95 p .
$$

Although not specifically labeled, the terms in Eq. (7) are obviously orientation dependent. We note that the absolute value of the surface dipole density, as defined by Eq. (3), is somewhat ambiguous since it depends on the starting point of integration $\left[z_{0}\right.$ in Eq. (3)]. This is compensated by the $V\left(z_{0}\right)$ in Eq. (7), which also depends on the choice of $z_{0}$. The difference gives the work function, which is independent of $z_{0}$ but dependent on orientation. In the numerical results shown below, the work function will be calculated with the usual procedure of comparing the Coulomb potential in the vacuum region with the Fermi level. While the absolute value of $p$, as defined as Eq. (3), depends on the choice of $z_{0}$, the adsorbate-induced change in dipole density can be found 
TABLE I. Calculated work functions for a monolayer of Mo, $\mathrm{Ag}, \mathrm{Au}, \mathrm{Fe}, \mathrm{Co}, \mathrm{Ni}, \mathrm{Nb}, \mathrm{Li}, \mathrm{N}$, and $\mathrm{O}$ on $\mathrm{W}(100), \mathrm{W}(110), \mathrm{W}(211)$, and $\mathrm{W}(111)$ surfaces. The unit cells are $p(1 \times 1)$. The first column (under Ref. 13) and the second column (under W) are the experimental and our calculated work functions of clean W surfaces in different orientations, respectively.

\begin{tabular}{lcccccccccccc}
\hline \hline & Ref. 13 & W & Mo & $\mathrm{Ag}$ & $\mathrm{Au}$ & $\mathrm{Fe}$ & $\mathrm{Co}$ & $\mathrm{Ni}$ & $\mathrm{Nb}$ & $\mathrm{Li}$ & $\mathrm{N}$ & $\mathrm{O}$ \\
\hline$(100)$ & 4.65 & 4.58 & 4.49 & 4.72 & 5.35 & 4.92 & 5.28 & 5.34 & 4.37 & 3.34 & 4.18 & 4.04 \\
$(110)$ & 5.25 & 5.18 & 4.97 & 4.84 & 5.34 & 4.73 & 4.72 & 4.74 & 4.84 & 3.30 & 6.64 & 6.19 \\
$(111)$ & 4.47 & 4.60 & 4.70 & 4.54 & 5.07 & 4.86 & 5.08 & 4.97 & 4.06 & 3.34 & 4.97 & 4.86 \\
$(211)$ & 4.76 & 4.75 & 4.55 & 4.78 & 5.18 & 5.05 & 5.17 & 5.15 & 4.15 & 3.40 & 5.35 & 4.84 \\
\hline \hline
\end{tabular}

unambiguously. When adsorbates are added, the surface dipole changes, which causes a change in $p$ and thus $\phi$, but the potential in the interior is unchanged. We can therefore write

$$
\phi-\phi_{0}=-180.95\left(p-p_{0}\right),
$$

where $\phi_{0}$ and $p_{0}$ are, respectively, the work function and the surface dipole of the clean surface. ${ }^{12}$ Equation (8) basically stated that the adsorbate induced change in the work function is proportional to the corresponding change in dipole moment density, and that there exists one single universal curve with a predetermined slope when $p-p_{0}$ is plotted against $\phi-\phi_{0}$.

\section{RESULTS}

We calculated the work function for $\mathrm{Mo}, \mathrm{Ag}, \mathrm{Au}, \mathrm{Fe}, \mathrm{Co}$, $\mathrm{Ni}, \mathrm{Nb}, \mathrm{Li}, \mathrm{N}$, and $\mathrm{O}$ on $\mathrm{W}(100), \mathrm{W}(110), \mathrm{W}(211)$, and $\mathrm{W}(111)$ surfaces, and the results are listed in Table I. The work function is calculated with the standard procedure, as illustrated in Fig. 1. We first obtained the atomic configurations corresponding to the relaxed atomic positions. We calculate the potential $\left[V(z)=V_{\text {local }}(z)+V_{H}(z)\right]$, where $V_{\text {local }}$ is the local part of the ionic pseudopotential and $V_{H}$ is the Hartree potential seen by the electrons. In the vacuum region, $V(z)$ is just the Coulomb potential. It is plotted as a function of $z$ and $V(z)$ typically goes to a flat and welldefined value rather quickly in the vacuum region, as shown in Fig. 1 for the case of W(110). The work function is simply the difference between $V(z)$ deep in vacuum and the Fermi level. We note that it is better to exclude the local exchangecorrelation potential $V_{x c}$, since it decays rather slowly in the

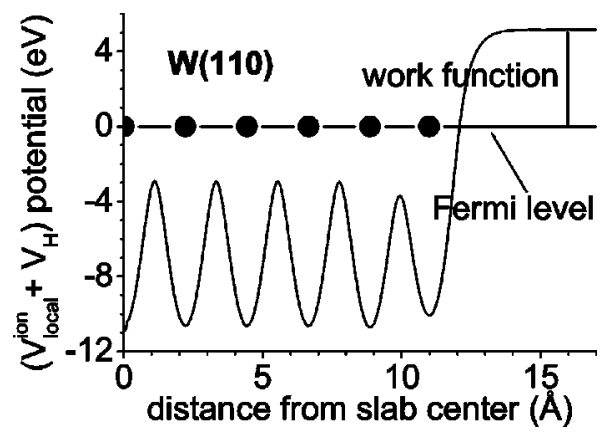

FIG. 1. Determination of work function by comparing the Fermi level with the local plus Coulombic potential in the vacuum. Solid circles mark the positions of $\mathrm{W}$ atoms in a W(110) slab. vacuum region and within the local-density formalism, the $V_{x c}$ has to be zero in the vacuum as charge density goes to zero there. There is no reason to include a slowly decaying part that would have mandated a bigger vacuum region in the repeated slab geometry.

The surface dipole is calculated according to Eq. (3), which just requires the total charge density and the atomic positions. The absolute value of the surface dipole density, as defined by Eq. (3), depends on the starting point of integration $\left[z_{0}\right.$ in Eq. (3)]. This is compensated by the $V\left(z_{0}\right)$ in Eq. (7), which also depends on the choice of $z_{0}$. The work function, as calculated with the "standard" procedure of comparing the Coulomb potential in vacuum with the Fermi level, is, of course, unambiguous as it does not involve any explicit integration. The adsorbate-induced change in dipole density is also unambiguous. In Fig. 2, we plot the absorbateinduced change in work function $\left(\Delta \phi=\phi-\phi_{0}\right)$ versus the absorbate-induced change in surface dipole density $(\Delta p=p$ $\left.-p_{0}\right)$.

We see from Fig. 2 that the absorbate-induced change in work function increases linearly with the absorbate-induced change in surface dipole density. All the numerical calculated values for a large number of adsorbates fall closely on one single straight line with a slope of 180.95 , as expected from Eq. (8). It should be emphasized that the work function and

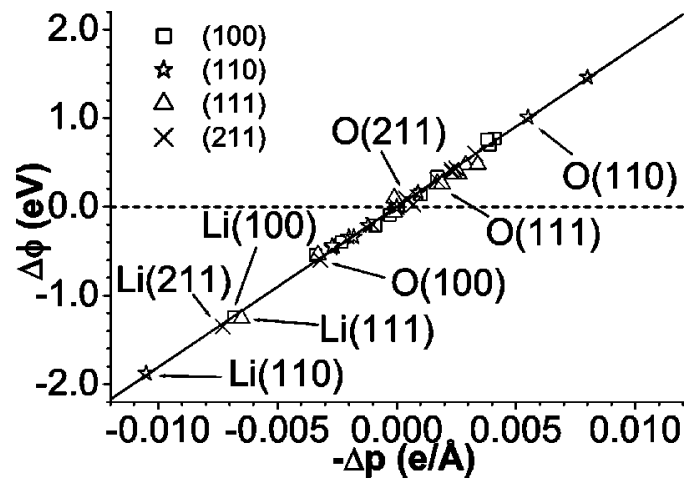

FIG. 2. Adsorbate induced change in work function plotted as a function of adsorbate induced change in surface dipole moment density for $\mathrm{Mo}, \mathrm{Ag}, \mathrm{Au}, \mathrm{Fe}, \mathrm{Co}, \mathrm{Ni}, \mathrm{Nb}, \mathrm{Li}, \mathrm{N}$, and $\mathrm{O}$ on $\mathrm{W}(100)$, $\mathrm{W}(110), \mathrm{W}(211)$, and $\mathrm{W}(111)$ surfaces. In order not to make the graph too crowded, we highlight the cases for $\mathrm{Li}$ and $\mathrm{O}$. The numerical values of the calculated work functions for other cases can be found in Table I. Note that Li always decreases the work function of the substrate but that the change is orientation dependent for O. 
dipole density shown in Fig. 2 are calculated independently, the former by comparing vacuum potential with the Fermi level and the latter by direct charge integration. This, together with the fact that the results for a large group of adsorbates are falling on one universal line in agreement with Eq. (8), provides a strong consistency check for all the calculations, and everything seems to be as expected. However, a careful look of the results reveal something interesting. For very electropositive adsorbates such as $\mathrm{Li}$, the work function is decreased substantially in all orientations. However, for most other materials, the trend is not directly followed. Examples are $\mathrm{Ni}, \mathrm{Co}$, and $\mathrm{Fe}$, which increase the work function of W(111), W(211), and W(100) (see Table I). This is expected because these metals are more electronegative than $\mathrm{W}$ on the Pauling scale. However, these metals decrease the work function on (110) (see Table I). Even oxygen and nitrogen, which are substantially more electronegative than W, do not always increase the work function when they are covering $\mathrm{W}$. We found that they increase the work function on (111), (211), and (110), but decrease the work function if they form a $(1 \times 1)$ layer covering $\mathrm{W}(100)$.

In order to better understand these observations, particularly that there are exceptions to the usual rule, we relate the work-function change induced by the adsorbate to the charge transfer and change of dipole moment. We calculate the change in the charge density due to the charge transfer from the substrate to the adsorbate, defined as

$$
\triangle \rho(z)=\rho(z)-\left[\rho_{s}(z)+\rho_{a}(z)\right],
$$

where $\rho(z)$ is the charge density of the adsorbate covered W surface, $\rho_{s}(z)$ is the charge density of the "substrate" W surface, which is generated by removing the overlayer of adsorbates from the coated $\mathrm{W}$ surface with all the $\mathrm{W}$ atoms frozen at the original position, and $\rho_{a}(z)$ is the charge density of the frozen overlayer, which is generated by removing all the $\mathrm{W}$ atoms from the slab unit cell, leaving the adsorbates behind. We can calculate the change of the dipole density due to the redistributed charge density by

$$
\triangle p=\int_{z_{0}}^{c / 2} z \triangle \rho(z) d z
$$

Substituting Eq. (9) into Eq. (10), we obtain

$$
\triangle p=p-\left(p_{s}+p_{a}\right),
$$

where $p_{s}$ is the surface dipole density of the $\mathrm{W}$ substrate and $p_{a}$ is the surface dipole density of the free-standing adsorbate layer. Due to the symmetry of the charge density of the freestanding overlayer, $p_{a}$ is equal to zero. We can rewrite Eq. (11) as

$$
p-p_{0}=\triangle p+\left(p_{s}-p_{0}\right),
$$

where $p_{0}$ is the surface dipole density of the clean $\mathrm{W}$ surface (fully relaxed). By using the relation between the dipole density and the work function as given by Eq. (8), we find that the change of the work function induced by the adsorbate is given by the following expression:

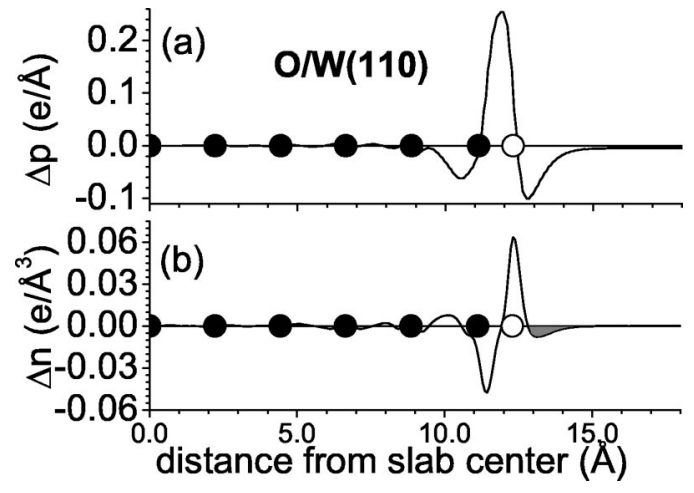

FIG. 3. (a) Adsorbate induced change in surface dipole moment density; and (b) electron density as a function of $z$ (see text for definition) for $\mathrm{O}$ on $\mathrm{W}(110)$.

$$
\phi-\phi_{0}=-\frac{e}{\varepsilon_{0}}\left[\triangle p+\left(p_{s}-p_{0}\right)\right]
$$

This equation shows that there are two contributions to the change of the work function induced by the adsorbates. The first is a charge rearrangement effect, contributed by the charge transfer due to the difference in electronegativity, and the hybridization between the adsorbate layer and the substrate, as given by $\triangle p$. The second contribution is due to the relaxation of the substrate induced by the overlayer, given by $\left(p_{s}-p_{0}\right)$. We find that the $\left(p_{s}-p_{0}\right)$ term is usually smaller than the $\triangle p$ term, and this is especially true for the most compact (110) orientation because the atomic relaxations are typically very small. The change of work function is essentially a chemical effect, and the change in atomic positions due to adsorbates can be taken as a secondary correction.

We now explain why the same adsorbate can increase the work function in one orientation, but decrease others, by considering two extreme cases of electronegativity: $\mathrm{O}$ and Li. Let us first consider the case of oxygen. Since O is significantly more electronegative than $\mathrm{W}$, we expect that when a layer of $\mathrm{O}$ atoms is adsorbed on $\mathrm{W}$ surface, electrons will be transferred from the $\mathrm{W}$ substrate to the $\mathrm{O}$ atoms, thereby increasing the work function. Our LDA calculation found that a layer of $\mathrm{O}$ atoms increases the work function when adsorbed on W(110), and also on (211) and (111), but decreases the work function on $\mathrm{W}(100)$. To see how this can happen, we plot two functions, $\triangle p(z)$ and $\triangle n(z)$, as a function of $z$ for the (110) and (100) orientations in Figs. 3 and 4, respectively. These functions are defined as

$$
\triangle p(z)=\int_{z_{0}}^{z} z^{\prime} \Delta \rho\left(z^{\prime}\right) d z^{\prime} .
$$

We note that $\Delta p(z) \rightarrow \Delta p$ [defined in Eq. (10)] as $z$ $\rightarrow c / 2$. The function $\Delta n(z)$ is the negative of $\Delta \rho(z)$, so that a negative value of $\Delta n(z)$ means a depletion of electrons and a positive value means an excess of electrons (negative charge).

In the figures, the center of the slab is defined as $z=0$. The solid dots mark the position of the $\mathrm{W}$ layers and the open circles mark the position of the adsorbate atoms. We 


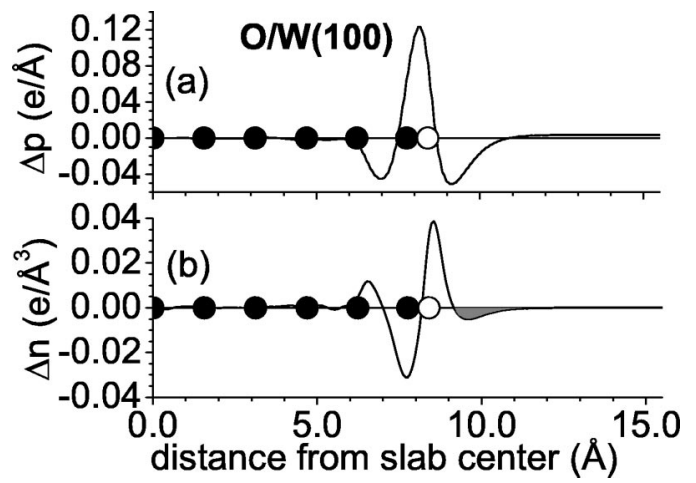

FIG. 4. (a) Adsorbate induced change in surface dipole moment density; and (b) electron density as a function of $z$ (see text for definition) for $\mathrm{O}$ on $\mathrm{W}(100)$.

see from Figs. 3 and 4 that in both cases, there is a depletion of electronic charge from the last $\mathrm{W}$ layer and an increase of electronic charge in the $\mathrm{O}$ layer, so that charge is indeed transferred from the $\mathrm{W}$ atoms to $\mathrm{O}$ atoms, in accordance with the electronegativity difference. Indeed, we found that the charge transfer is basically dictated by the electronegativity scale, and charge is always transferred from $\mathrm{W}$ to $\mathrm{O}$, independent of orientation. However, the change in dipole density $\Delta p(z)$ as a function of $z$ is more subtle and is orientation dependent. We note from Eq. (10) that the dipole is weighted by the distance from the center of the slab. We see from both the $\Delta n$ plots Figs. 3 and 4, that while the oxygen atoms gain electrons from the $\mathrm{W}$, there is also a depletion of electrons in the outer "tail" region of the oxygen layer, as marked by the shaded areas in the (b) panels of Figs. 3 and 4. This is due to the rearrangement of electronic charge density in the oxygen layer to form chemical bonding in the W-O interface. The number of electrons inside the shaded area is small, so that it will not affect the sign of charge transfer. However, when weighted by " $z$," this can have important consequences on the sign of $\Delta p$. If the shaded area in the $\Delta n$ plots were zero, the value of $\Delta p$ is negative for both $\mathrm{O}$ on $\mathrm{W}(110)$ and $\mathrm{W}(100)$, which would then have increased the work function for both orientations. However, the depletion of electrons in the shaded region gives a positive contribution to $\Delta p$. Although the amount of charge in the shaded region is small, the weighting by $z$ in the integral [see Eq. (10)] may be sufficient to turn the sign of $\Delta p$ from negative to positive for the (100) orientation, which then makes the work function lower than that of the clean surface. For the (110) and other orientations we have studied, the positive contribution from the shaded area in the $\Delta n$ plot is not big enough to change the sign of $\Delta p$, and thus the work function remains higher than that of the clean surface, a result that would have been expected for consideration of charge transfer. This picture is qualitatively the same for other elements such as $\mathrm{N}$, which are significantly more electronegative than W. For the (100) orientation, we can see in Fig. 4 that the distance from the outermost $\mathrm{W}$ layer to the adsorbate $\mathrm{O}$ layer is quite small. In such a situation, the charge transfer from $\mathrm{W}$ to $\mathrm{O}$ is not effective in giving rise to the surface dipole, and thus the contribution of the tail is important in this case.

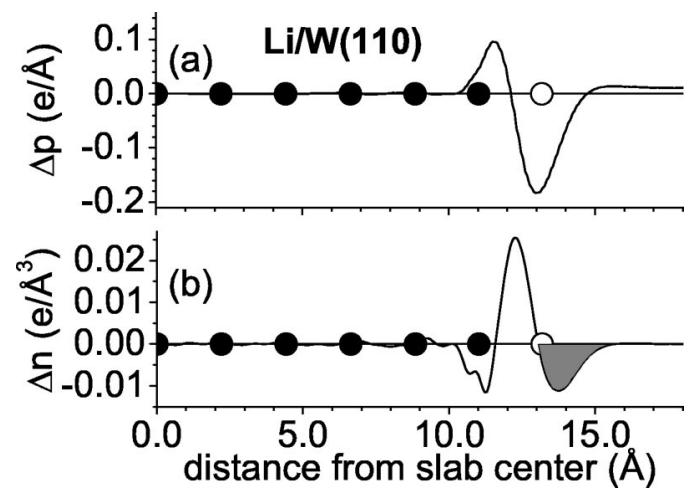

FIG. 5. (a) Adsorbate induced change in surface dipole moment density; and (b) electron density as a function of $z$ (see text for definition) for $\mathrm{Li}$ on $\mathrm{W}(110)$.

For adsorbates such as $\mathrm{Li}$ that are very electropositive relative to the substrate, the situation is somewhat simpler. We found that there is always a charge transfer from the more electropositive $\mathrm{Li}$ atoms to the interlayer region between $\mathrm{W}$ and $\mathrm{Li}$, independent of the substrate orientation. This can be seen in Figs. 5(b) and 6(b). The tail region in the outer Li layers, marked as the shaded area in Figs. 5(b) and 6(b), is a depletion of electrons. This contribution to $\Delta p$ is always positive, independent of orientations, and has the same sign as would have been predicted from electronegativity arguments, leading to the same sign of $\Delta p$ as $z \rightarrow c / 2$ for both the (110) and (100) orientations as seen in Figs. 5(a) and 6(a). Thus there is a decrease of work function in all orientations.

For the cases in which the adsorbates are metals that have comparable Pauling electronegativity with the $\mathrm{W}$ substrate, the change in work function is usually orientation dependent. Examples are $\mathrm{Ag}, \mathrm{Fe}, \mathrm{Co}, \mathrm{Ni}$, and $\mathrm{Mo}$, as can be seen in Table I. Here, the bonding is essentially metallic and the change in charge density is more complex. The increase or decrease in work function depends on the fine details of the charge rearrangement.

We note that all the above calculations are performed with LDA and ultrasoft pseudopotentials. Since the values of the surface dipole and the work function depend on the details on the charge density, we also check some of our results

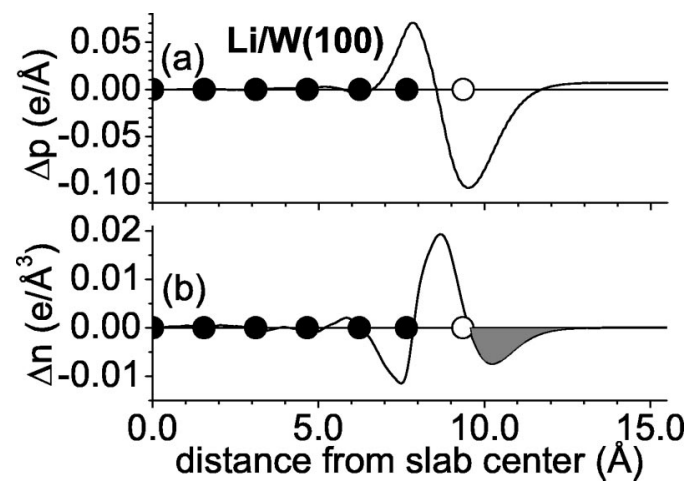

FIG. 6. (a) Adsorbate induced change in surface dipole moment density; and (b) electron density as a function of $z$ (see text for definition) for $\mathrm{Li}$ on $\mathrm{W}(100)$. 
using the Perdew-Burke-Ernzerhof(PBE) form of generalized gradient approximation (GGA) (Ref. 14) and the projected augmented wave (PAW) method. ${ }^{15}$ The work functions calculated for clean $\mathrm{W}(100)$, clean $\mathrm{W}(110), \mathrm{O} / \mathrm{W}(100)$, $\mathrm{O} / \mathrm{W}(110), \mathrm{Li} / \mathrm{W}(100)$, and $\mathrm{Li} / \mathrm{W}(110)$ are 4.10, 4.78, 3.90, 5.87, 3.12, and $3.21 \mathrm{eV}$, respectively. Comparing with the LDA results listed in Table I, we see that the PBE calculated work functions are always lower than those of the LDA. However, the qualitative results are the same. For example, both LDA and GGA found that the work function of W(100) is lower than that of $\mathrm{W}(110)$, and that $\mathrm{Li}$ lowers the work function of $\mathrm{W}$ significantly in all orientations. In particular, both LDA and GGA found that $\mathrm{O}$ increases the work function of $\mathrm{W}(110)$ but lowers that of $\mathrm{W}(100)$, and thus the anomaly we found is independent of the form of the exchange-correlation used. The curves of $\triangle p(z)$ and $\triangle n(z)$ calculated from GGA-PBE and PAW are qualitatively the same as those shown in Figs. 3-6. Thus, the details of the exchange correlation and the potential will not affect the results.

\section{SUMMARY}

We have calculated the work function and surface dipoles for a large number of elements adsorbed on various orientations of $\mathrm{W}$ substrates and demonstrated the simple relation between the work function and the dipole density. The standard electronegativity scale can predict reliably the direction of charge transfer but not necessarily the adsorbate induced change in work function. Some explicit examples are analyzed in detail to show that work-function changes are not just decided by the quantity and the sign of charge transferred, but also by the details of the charge redistribution, leading in some cases to a strong dependence of the workfunction change on the orientation of the substrate.

\section{ACKNOWLEDGMENTS}

This work was supported by Grant No. (NSC)91-2112-M194-023 and by RGC Hong Kong through Grant No. HKUST6152/01P. Computation resources were supported by a grant of computer time at the National Center for HighPerformance Computing, and an EHIA grant from HKUST.
${ }^{1}$ E. Wigner and J. Bardeen, Phys. Rev. 48, 84 (1935).

${ }^{2}$ N.D. Lang and W. Kohn, Phys. Rev. B 3, 1215 (1971).

${ }^{3}$ R. Smoluchowski, Phys. Rev. 60, 661 (1941).

${ }^{4}$ P.J. Goddard and R.M. Lambert, Surf. Sci. 67, 180 (1977).

${ }^{5}$ V.M. Gavrilyuk and V.K. Medvedev, Sov. Phys. Solid State 8, 1439 (1966).

${ }^{6}$ R.O. Jones and O. Gunnarsson, Rev. Mod. Phys. 61, 689 (1989).

${ }^{7}$ See, for example, H.L. Skriver and N.M. Rosengaard, Phys. Rev. B 4, 7157 (1992); M. Methfessel, D. Hennig, and M. Scheffler, ibid. 46, 4816 (1992); E. Wimmer, A.J. Freeman, J.R. Hiskes, and A.M. Karo, ibid. 28, 3074 (1983); N. Wang, K. Chen, and D.S. Wang, Phys. Rev. Lett. 56, 2759 (1986); R.Q. Wu, K. Chen, D.S. Wang, and N. Wang, Phys. Rev. B 38, 3180 (1988).

${ }^{8}$ P. Hohenberg and W. Kohn, Phys. Rev. 136, B864 (1964); W. Kohn and L.J. Sham, ibid. 140, A1133 (1965).

${ }^{9}$ G. Kresse and J. Hafner, Phys. Rev. B 47, 558 (1993); 49, 14251 (1994); G. Kresse and J. Furthmuller, Comput. Mater. Sci. 6, 15 (1996); Phys. Rev. B 54, 11169 (1996).

${ }^{10}$ G. Kresse and J. Hafner, J. Phys.: Condens. Matter 6, 8245 (1994).

${ }^{11}$ In the experimental literature, a monolayer on the bcc (111) ori- entation sometimes corresponds to a "physical monolayer" rather than a geometric monolayer. A physical monolayer of adsorbates is such that the overlayer shadows all the substrate atoms in a ball-and-stick model of the substrate, while a geometric monolayer is an additional layer of the truncated bulk. A physical monolayer may contain more atoms than a geometric monolayer in open surfaces like (111) surface of bcc metals. For details, see, for example, T.E. Madey, J. Guan, C.H. Nien, C.Z. Dong, H.-S. Tao, and R.A. Campbell, Surf. Rev. Lett. 3, 1315 (1996).

${ }^{12}$ Equation (7) requires that we choose $z_{0}$ where $\partial V / \partial z=0$. However, when we calculate the difference of dipole moments in Eq. (8), the choice of $z_{0}$ can be arbitrary as long as it is deep inside the bulk, since the terms originating from the integration starting point defined in Eq. (3) always cancel out.

${ }^{13}$ Y. Yamamoto and T. Miyokawa, J. Vac. Sci. Technol. B 16, 2871 (1998).

${ }^{14}$ J.P. Perdew, K. Burke, and M. Ernzerhof, Phys. Rev. Lett. 77, 3865 (1996).

${ }^{15}$ P.E. Blöchl, Phys. Rev. B 50, 17953 (1994). 